\title{
Achievement of Long-term Local Control After Concurrently With Radiation and Anti-PD-1 Immunotherapy in Locally Advanced Non-small Cell Lung Cancer Patients
}

\section{zhao jing ( jz96329@163.com )}

Department of Radiation Oncology, Hangzhou Cancer Hospital, Hangzhou; 34 Yanguan Lane, Hangzhou 310002, Zhejiang 310002, P. R. China. https://orcid.org/0000-0002-6566-599X

Rongjin Zhou

hangzhou cancer hospital

Huaxiang He

hangzhou cancer hospital

Shixiu Wu

cancer hospital shenzhen hospital

\section{Case report}

Keywords: Non-small cell lung cancer, Checkpoint inhibitor, Immunotherapy, Radiotherapy,

Pembrolizumab

Posted Date: September 8th, 2020

DOI: https://doi.org/10.21203/rs.3.rs-72750/v1

License: (c) (i) This work is licensed under a Creative Commons Attribution 4.0 International License.

Read Full License 


\section{Abstract}

Background: Although concurrent chemoradiotherapy (CRT) was recommend as standard of care in patients with stage III unresectable non-small cell lung cancer (NSCLC), many patients refused or were not eligible for chemotherapy in clinical practice. These patients often receive RT alone with 5-year OS rate of about $5-6 \%$. This addressed a common clinical challenge of treating these patients. Immunecheckpoint inhibitors have demonstrated objective antitumor responses in patients with advanced NSCLC, but it is unclear how these agents can be used in the curative therapy with concurrent radiation.

Case presentation: Here we described, the case series, the effect of stage III unresectable NSCLC patients who refused chemotherapy received radiation and anti-PD-1 immunotherapy. Three patients with stage III unresectable NSCLC were treated with radiotherapy concurrently with anti-PD-1 agent (pembrolizumab) between May 2018 and August 2018 in our hospital. Two patients experienced partial response and one patient experienced stable disease. One patient developed the liver metastasis 4 months after the treatment. All patients had no local-regional recurrence. No patient experienced $\geq$ grade 3 adverse event $(\mathrm{AE})$, and no patient discontinued treatment because of an $\mathrm{AE}$.

Conclusions: Concurrent treatment with radiation and pembrolizumab for unresectable stage III NSCLC patients who refused chemotherapy demonstrated its efficacy and acceptable tolerance. Further investigations are warranted to determine its role in the management of these patients.

\section{Background}

Lung cancer is the leading cause of cancer-related incidents and mortality worldwide. Approximately $30 \%$ non-small-cell lung cancer (NSCLC) patients are diagnosed as stage III (locally advanced), unresectable NSCLC. Concurrent platinum-based chemoradiotherapy was recommend as standard of care in patients with stage III unresectable NSCLC. ${ }^{1,2}$ However, many patients refused or were not eligible for chemotherapy in clinical practice. Some studies reported that about $50 \%$ patients with stage III lung cancer were ineligible for concurrent chemoradiotherapy (CRT). ${ }^{3,4}$ For the patients who refused or were not eligible for chemotherapy often receive RT alone with a 5 -year OS rate of about $5-6 \% .{ }^{5}$ This addressed a common clinical challenge of treating these patients. New therapeutic approaches are needed to improve survival for these patients.

Immunotherapy has changed treatment paradigms in recent years. Immunotherapy targeting the PD1/PD-L1 axis has developed new potential strategies for treatment of patients with advanced NSCLC. Although some patients achieve durable responses to checkpoint blockade, most patients do not experience such benefits. The growing evidence support the combination of immunotherapy with chemotherapy and radiation. Preclinical evidences have demonstrated that combining radiotherapy with anti-PD-1 treatment could enhance the anti-tumor activity and produce long-term survival. The PACIFIC trial showed that anti-PD-L1 (durvalumab) as consolidation therapy significantly prolonged progressionfree survival (PFS) and OS in patients with stage III, unresectable NSCLC. ${ }^{6}$ 
Immune checkpoint inhibitors have improved outcomes mainly in patients with advanced NSCLC, but it is unclear how these agents can be used in the curative setting concurrent with radiation. Herein, we present three patients with stage III unresectable NSCLC who refused chemotherapy received radiation concurrently with anti-PD-1 agent (pembrolizumab).

\section{Case Presentation}

A total of 3 patients with unresectable stage III NSCLC who refused chemotherapy received radiation concurrently with pembrolizumab between May 2018 and August 2018 in our hospital. The pembrolizumab was administered at a fixed dose $200 \mathrm{mg}$ starting on day 1 and given every 21 days for up to 5 cycles. Intensity-modulated radiation therapy (IMRT) started on the first day of pembrolizumab. Conventionally fractionated radiation therapy delivered a total dose of 60 Gy given in 30 fractions over 6 weeks. PD-L1 expression was immunohistochemically assessed with the use of the Dako 22C3 in the archived tumor tissue specimens obtained before treatment. Follow-up scans with CT, or PET/CT were obtained every 12 weeks ( \pm 4 weeks) to evaluate response to therapy. The tumor response was assessed according to the Response Evaluation Criteria in Solid Tumors (RECIST) version 1.1. Adverse events and abnormal laboratory findings were graded according to the National Cancer Institute Common Terminology Criteria for Adverse Events (CTCAE) version 4.0. All patients gave their informed consent prior to their inclusion in the study.

\section{Case 1}

A 61-year-old male former smoker initially presented in May 2018 with one month of cough and neck pain. Chest computed tomography (CT) revealed a $3.5 \times 3.0 \mathrm{~cm}$ left lung mass (Figure $1 \mathrm{~A}$ ) as well as mediastinal, and supraclavicular lymphadenopathy. Brain magnetic resonance imaging (MRI) was normal. Biopsies of the supraclavicular masses showed lung squamous cell carcinoma (Figure 1B). No mutations were detected for EGFR or ALK. The PD-L1 expression (tumor proportion score, TPS) was negative (Figure 1C). According to the American Joint Committee on Cancer (AJCC) staging manual (8th ed), his disease was characterized as stage IIIB (T2N3M0) and was therefore considered inoperable. The patient had an Eastern Cooperative Oncology Group (ECOG) score of 1 and adequate organ and pulmonary function. The patient initially refused chemotherapy. Thus, the combination of local radiation and pembrolizumab was recommended. The treatment was well tolerated. The patient experienced grade 2 radiation pneumonitis and grade 1 esophagitis. One month after the treatment, the patient was deemed to show a partial response (PR) on June 26,2018 . There was a $70 \%$ local tumor reduction based on contrast-enhanced CT analysis (Figure 1A). Over the course of 24 months, the patient developed longterm disease stabilization and is alive at his last visit without local recurrence or metastasis (July 2020).

\section{Case 2}

A 59-year-old never-smoking male initially presented in June 2018 with several months of persistent cough. Chest computed tomography (CT) revealed a $4.5 \times 3.3 \mathrm{~cm}$ right lung mass (Figure $2 \mathrm{~A}$ ) as well as mediastinal lymphadenopathy. Brain magnetic resonance imaging (MRI) was normal. Biopsies of the 
lung showed lung squamous cell carcinoma (Figure 2B). No mutations were detected for EGFR or ALK. The PD-L1 expression (TPS) was negative (Figure 2C). According to the AJCC staging manual (8th ed), his disease was characterized as stage IIIA (T2N2M0) and was therefore considered inoperable. The patient had an Eastern Cooperative Oncology Group (ECOG) score of 1 and adequate organ and pulmonary function. The patient initially refused chemotherapy. Thus, he received the combination of local radiation with pembrolizumab. One month after the combination therapy, the patient was deemed to show a stable disease (SD) on July 11, 2018 (Figure 2A). He experienced grade 2 radiation pneumonitis. This SD was maintained in less than 4 months, and the patient developed a new liver metastasis. Treatment consisted of radiofrequency ablation of the liver metastasis, followed by 6 cycles nabpaclitaxel ( $400 \mathrm{mg} / \mathrm{m}^{2}$, each 3-week cycle). After completing treatment in March 2019, the patient developed 16 months disease stabilization and is alive at his last visit without local recurrence or any other metastasis. (July 2020).

\section{Case 3}

A 59-year-old former smoker male initially presented in August 2018 with one months of progressive dyspnea, and persistent cough. Chest computed tomography (CT) revealed a $5.5 \times 3.6 \mathrm{~cm}$ right hilar mediastinum mass (Figure 3A). Brain magnetic resonance imaging (MRI) was normal. Bronchoscopy biopsy of the lung showed lung squamous cell carcinoma (Figure 3B). No mutations were detected for EGFR or ALK. The PD-L1 expression (TPS) was 30\% (Figure 3C). According to the AJCC staging manual (8th ed), his disease was characterized as stage IIIB (T3N2M0) and was therefore considered inoperable. The patient had an Eastern Cooperative Oncology Group (ECOG) score of 1 and adequate organ and pulmonary function. The patient initially refused chemotherapy. The patient received the combination of

local radiation with pembrolizumab. One month after the combination therapy, the patient was deemed to show a PR on September 16, 2018. There was a $80 \%$ local tumor reduction based on contrast-enhanced CT analysis (Figure $3 \mathrm{~A}$ ). The treatment were tolerated without serious adverse effects. The patient experienced grade 1 fatigue. The patient continued to be in stabilization for 22 months up to the last follow-up without local recurrence or metastasis (July 2020).

\section{Discussion And Conclusions}

Many patients with stage III lung cancer were ineligible for concurrent CRT, medically unsuitable or refused to chemotherapy. The prognosis of these patients received RT alone was poor. Immune checkpoint blockade is an exciting treatment option for cancer. It has been demonstrated that immune checkpoint inhibitors and radiation had a synergistic effect. The mechanisms of radiation enhancing immunotherapy include increased tumor antigen release, augmented antigen presentation, activation of innate immune pathways, increased T-cell infiltration, and modulation of immunosuppressive cells. 7,8 The safety and efficacy of this combination have also been confirmed in metastatic solid tumors. But it is still unclear how this combination can be used in the curative setting. 
The case series demonstrating the effect of curative radiation and anti-PD- 1 immunotherapy in patients with stage III unresectable NSCLC. Limited by the short follow-up time and small sample size, we still found the potential effect of this treatment. All patients had no local-regional recurrence. The case series only received 5 cycles of pembrolizumab treatment, but the responses maybe long-lasting. The efficacy of immunotherapy in which a significant tumor regression would translate into extension of overall survival. Previous research have demonstrated the synergistic efficacy of high-dose fractionated radiotherapy combined with anti-PD- 1 therapy ${ }^{16}$. Current data from our experience showed conventionally fractionated radiotherapy also has synergistic effect with anti-PD-1 antibody. Due to lack of direct comparison, the optimal fraction scheme in combination with anti-PD-L1 therapy needs further investigation. This case series may play an important role for future clinical trial design, since most patients received conventionally fractionated radiotherapy in clinical practice.

Although the CRT was the standard treatment of NSCLC patients with stage III, the treatment toxicity was obvious, with $42 \%$ grade 3 acute toxicities, $25 \%$ grade 3 late toxicities ${ }^{1}$. About $20 \%$ patients could not complete the concurrent treatment. In the present case series, the pembrolizumab can be safely added to radiotherapy without an increase in the toxicity profiles. No patient experienced $\geq$ grade $3 \mathrm{AE}$, and no patient discontinued treatment because of an $\mathrm{AE}$.

Immunohistochemistry assay for PD-L1 expression remains the current standard for identifying patients with NSCLC who are more likely to benefit from immunotherapy ${ }^{9}$. Many prospective trials have demonstrated a correlation between the level of tissue PD-L1 expression and clinical efficacy. However, not all patients with high PD-L1 expression respond to therapy, and some patients with low or no expression of PD-L1 also achieve benefit. In this case series, the Case \#1 patient with no expression of PD-L1 still demonstrate a durable clinical benefit from the treatment of pembrolizumab plus radiation. Currently, PD-L1 still remains a controversial biomarker with some limitations: differences in testing platforms, the use of different cutoff points for different immunotherapy agents, and the heterogenous expression within tumors. Compared with monotherapy, it is more difficult to identify which patients may derive the benefit from combined immunotherapy. In addition to PD-L1 expression, high microsatellite instability (MSI), DNA mismatch repair deficiency (dMMR), and high tumor mutational burden (TMB) also approved as biomarkers of immunotherapy ${ }^{10}$. Clinical studies with larger samples are required to develop suitable biomarkers for predicting toxicity and sensitive of radiation plus immunotherapy response.

\section{Conclusions}

We provided the case series of combination of curative radiation with pembrolizumab for unresectable stage III NSCLC patients who refuse chemotherapy. Although there may be different immune backgrounds and biomarkers of immune response in the three patients, we successfully treated them with radiation and the PD-1 inhibitor pembrolizumab. All patients achieved long-term local control with acceptable tolerance. Further investigations are warranted to determine its role in the management of stage III unresectable NSCLC patients who refused or were ineligible for chemotherapy. 


\section{Abbreviations}

$A E$, adverse event; $C T$, computed tomography; dCRT, definitive concurrent chemoradiotherapy; dMMR, DNA mismatch repair deficiency; ECOG, Eastern Cooperative Oncology Group; GTV, gross tumor volume; IMRT, Intensity-modulated radiation therapy; MSI, microsatellite instability; NSCLC, non-small cell lung cancer; OS, overall survival; PD-1, Programmed death 1; PD-L1, Programmed death ligand 1; PET, positronemission tomography; PFS, progression-free survival; PR, partial response; SD, stable disease; TMB, Tumor mutational burden; TPS, Tumor proportion score

\section{Declarations}

\section{Acknowledgements}

Not applicable.

\section{Conflict of interest}

The author declare that they have no competing interest.

\section{Authors' contributions}

$\mathrm{JZ}$ and $\mathrm{HH}$ carried out the literature search and image and data collection, and drafted the article. ZR collected important background information and performed image processing. manuscript; WS revised the manuscript. All authors read and approved the final manuscript.

\section{Funding}

None.

\section{Availability of data and materials}

Not applicable.

\section{Consent for publication}

Not applicable.

\section{Ethics approval and consent to participate}

All procedures performed in present study were in accordance with the ethical standards. The informed consent was obtained for each patient before any medical act is performed.

\section{Author details}


${ }^{1}$ Department of Radiation Oncology, ${ }^{2}$ Department of Pathology, ${ }^{3}$ Department of nursing, Hangzhou Cancer Hospital, Hangzhou, Zhejiang, PR China

${ }^{3}$ Department of Radiation Oncology, National Cancer Center/National Clinical Research Center for Cancer/Cancer Hospital \& Shenzhen Hospital, Chinese Academy of Medical Sciences and Peking Union Medical College, Shenzhen, Guangdong, PR China

\section{References}

1. Aupérin $A$, Le Péchoux $C$, Rolland E, et al. Meta-analysis of concomitant versus sequential radiochemotherapy in locally advanced non-small-cell lung cancer. J Clin Oncol. 2010; 28(13): 218190.

2. Liang J, Bi N, Wu S, et al. Etoposide and cisplatin versus paclitaxel and carboplatin with concurrent thoracic radiotherapy in unresectable stage III non-small cell lung cancer: a multicenter randomized phase III trial. Ann Oncol. 2017; 28(4): 777-83.

3. De Ruysscher D, Botterweck A, Dirx M, et al. Eligibility for concurrent chemotherapy and radiotherapy of locally advanced lung cancer patients: a prospective, population-based study. Ann Oncol. 2009; 20(1): 98-102.

4. Al-Shamsi HO, Al Farsi A, Ellis PM. Stage III non-small-cell lung cancer: Establishing a benchmark for the proportion of patients suitable for radical treatment. Clin Lung Cancer. 2014; 15(4): 274-80.

5. Sonnick MA, Oro F, Yan B, et al. Identifying the Optimal Radiation Dose in Locally Advanced NonSmall-cell Lung Cancer Treated With Definitive Radiotherapy Without Concurrent Chemotherapy. Clin Lung Cancer. 2018; 19(1): e131-e140.

6. Antonia SJ, Villegas A, Daniel D, et al. Overall Survival with Durvalumab after Chemoradiotherapy in Stage III NSCLC. N Engl J Med. 2018; 379(24): 2342-50.

7. Demaria S, Golden EB, Formenti SC. Role of Local Radiation Therapy in Cancer Immunotherapy. JAMA Oncol. 2015; 1(9): 1325-32.

8. Bates JE, Morris CG, Milano MT, Yeung AR, Hoppe BS. Immunotherapy with hypofractionated radiotherapy in metastatic non-small cell lung cancer: An analysis of the National Cancer Database. Radiother Oncol. 2019; 138: 75-79.

9. Roach C, Zhang N, Corigliano E, et al. Development of a Companion Diagnostic PD-L1 Immunohistochemistry Assay for Pembrolizumab Therapy in Non-Small-cell Lung Cancer. Appl Immunohistochem Mol Morphol. 2016; 24(6): 392-7.

10. Overman MJ, McDermott R, Leach $\mathrm{JL}$, et al. Nivolumab in patients with metastatic DNA mismatch repair-deficient or microsatellite instability-high colorectal cancer (CheckMate 142): an open-label, multicentre, phase 2 study. Lancet Oncol. 2017; 18(9): 1182-91.

\section{Figures}


Figure 1

$\mathbf{A}$
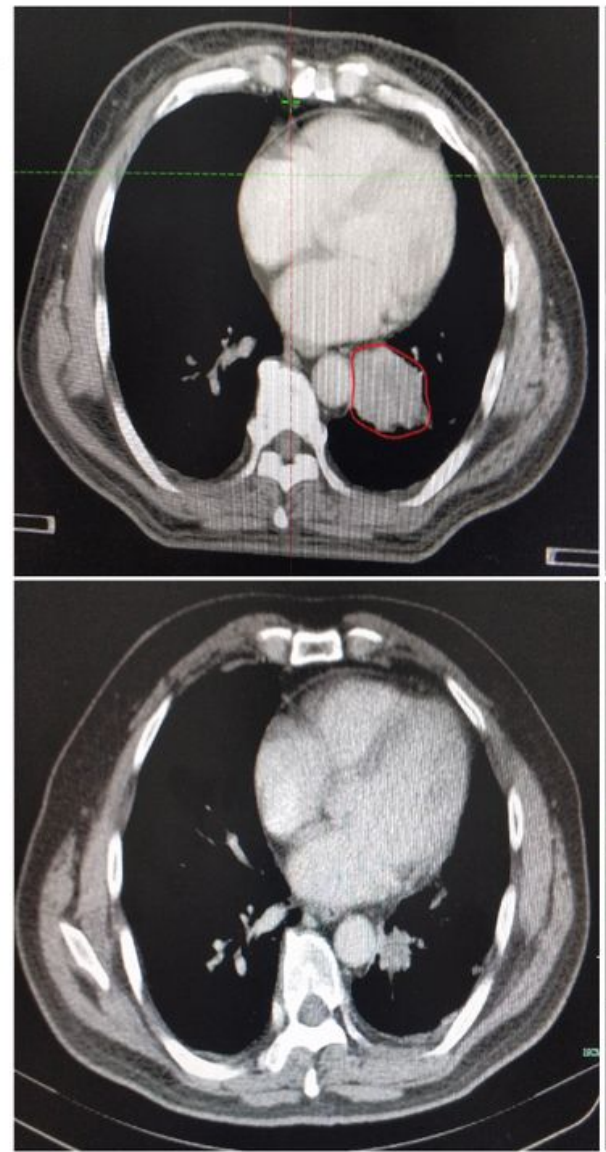

Baseline
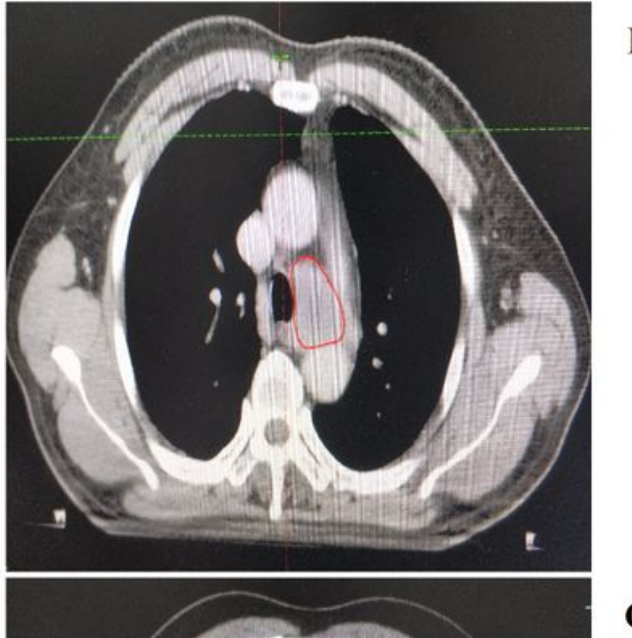

C
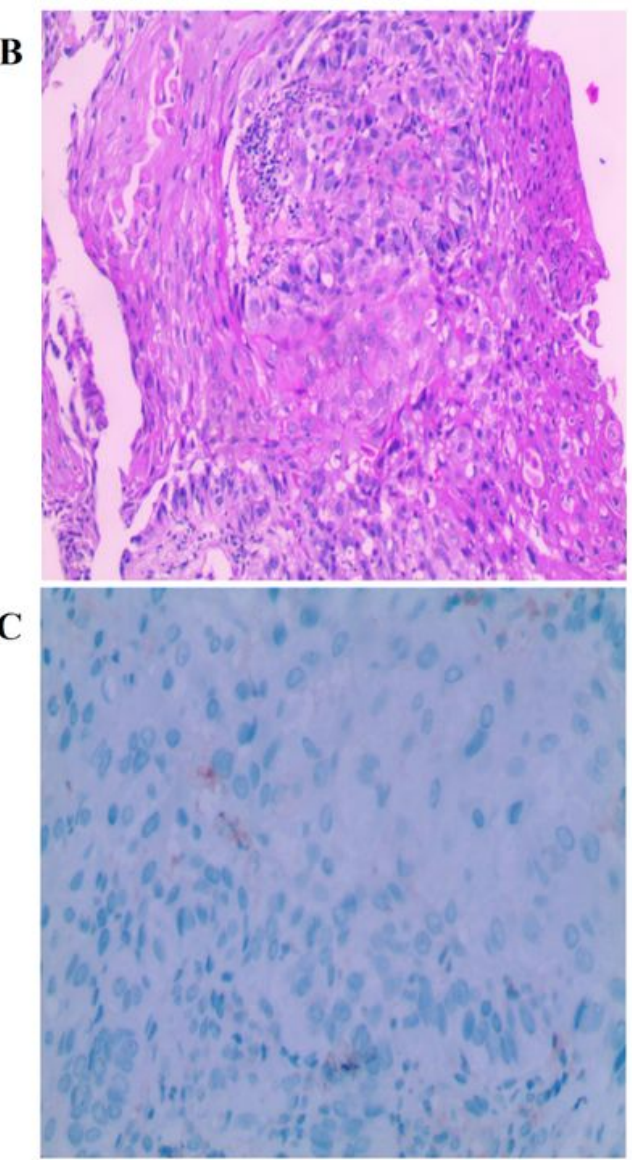

Best response

Figure 1

(Patient 1) A. Contrast-enhanced chest CT prior and best response to treatment. B. Hematoxylin and eosin-stained section of lymph node biopsy specimen. C. PD-L1 protein expression assessed by immunohistochemistry. 
Figure 2

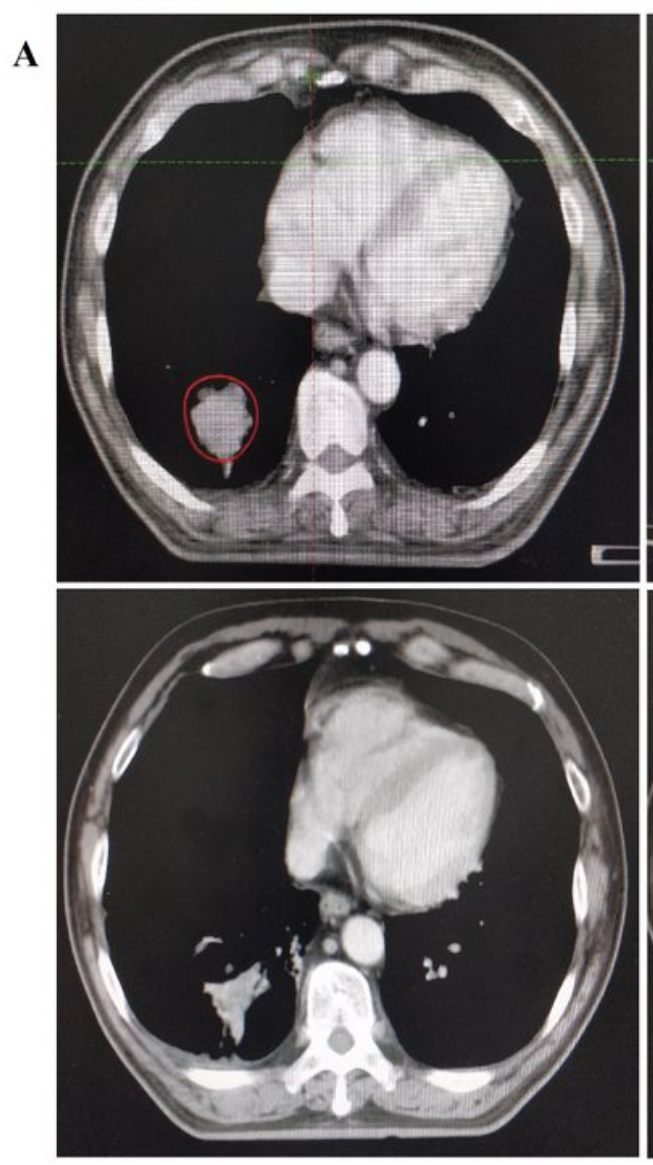

Baseline
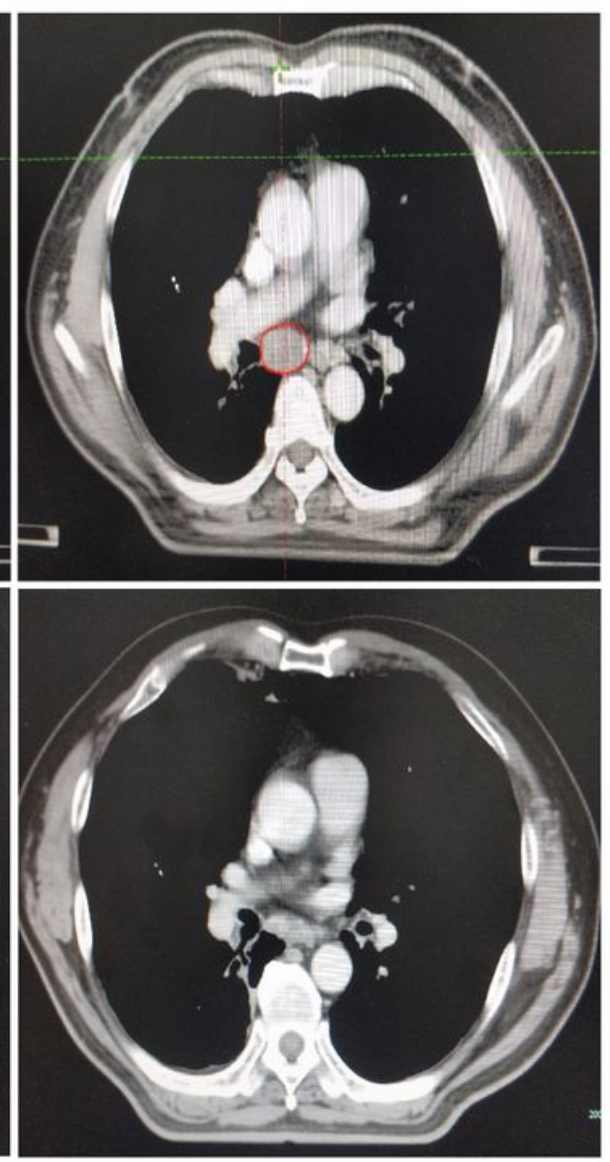

Best response

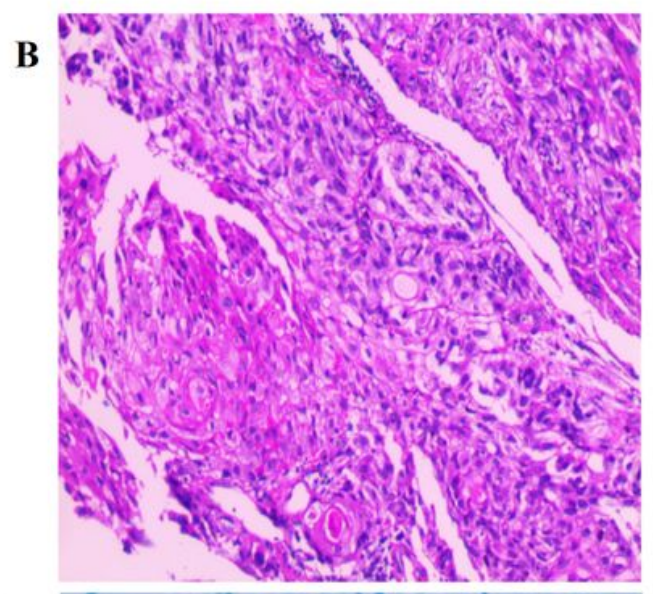

C

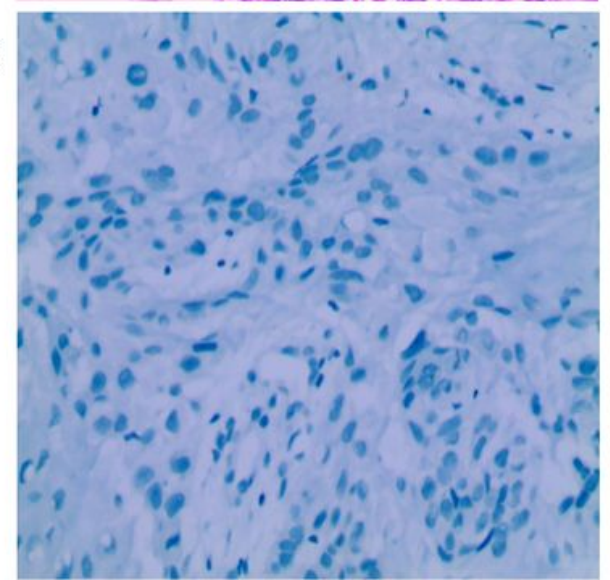

\section{Figure 2}

(Patient 2) A. Contrast-enhanced chest CT prior and best response to treatment. B. Hematoxylin and eosin-stained section of lung lesion biopsy specimen. C. PD-L1 protein expression assessed by immunohistochemistry. 
Figure 3
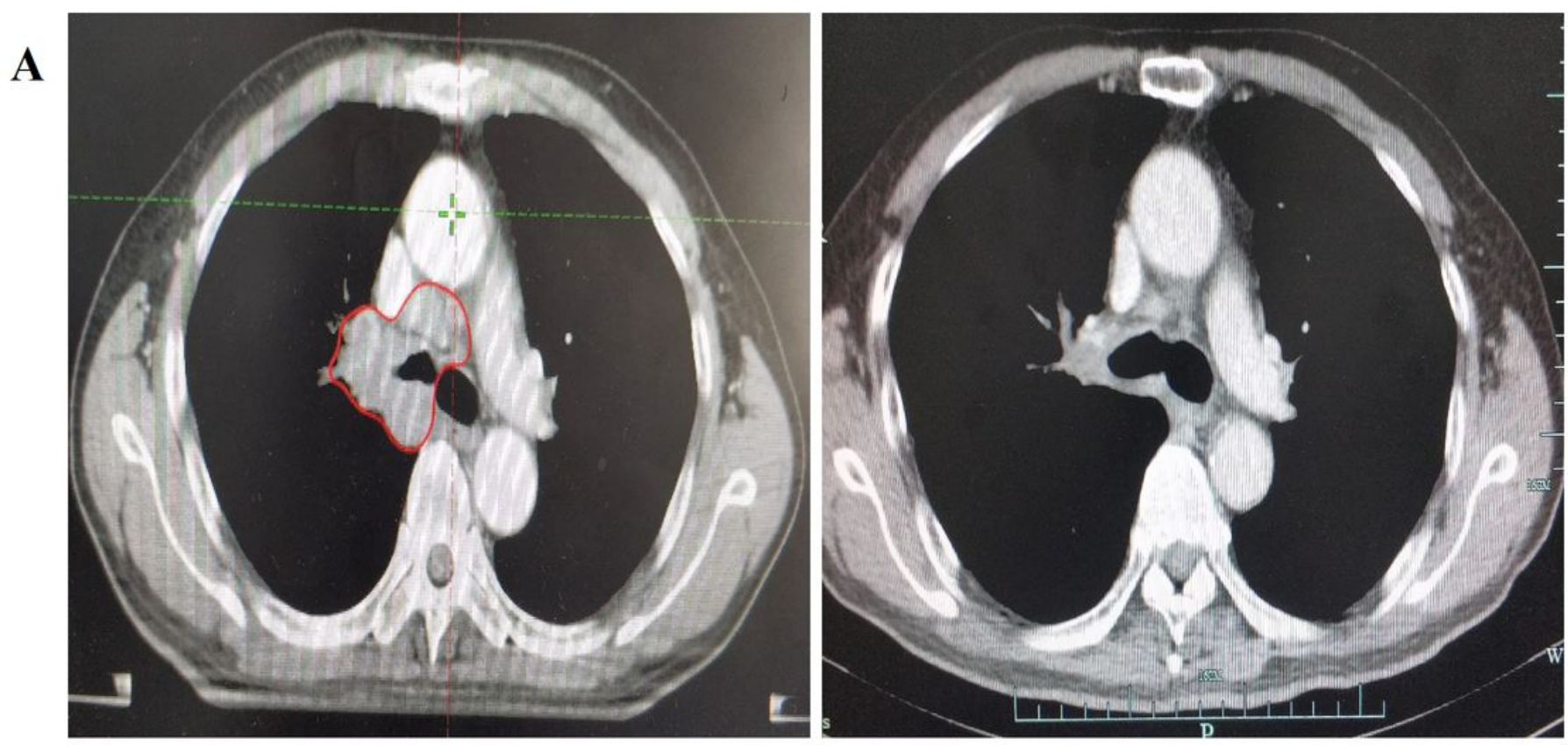

\section{Baseline}

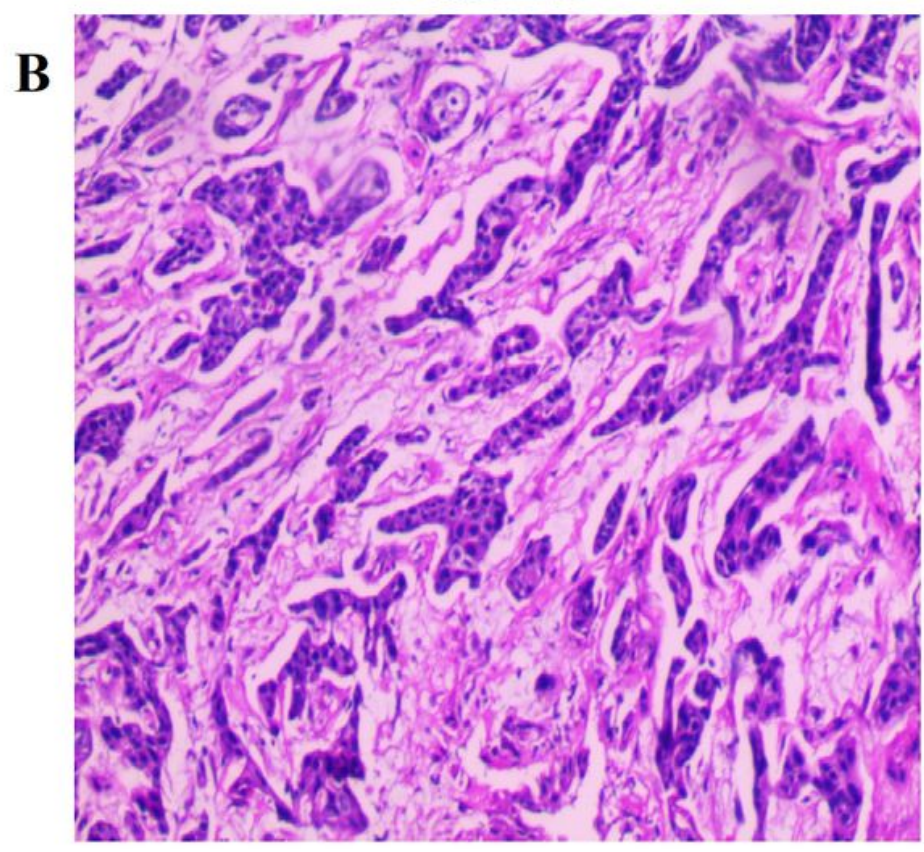

\section{Best response}

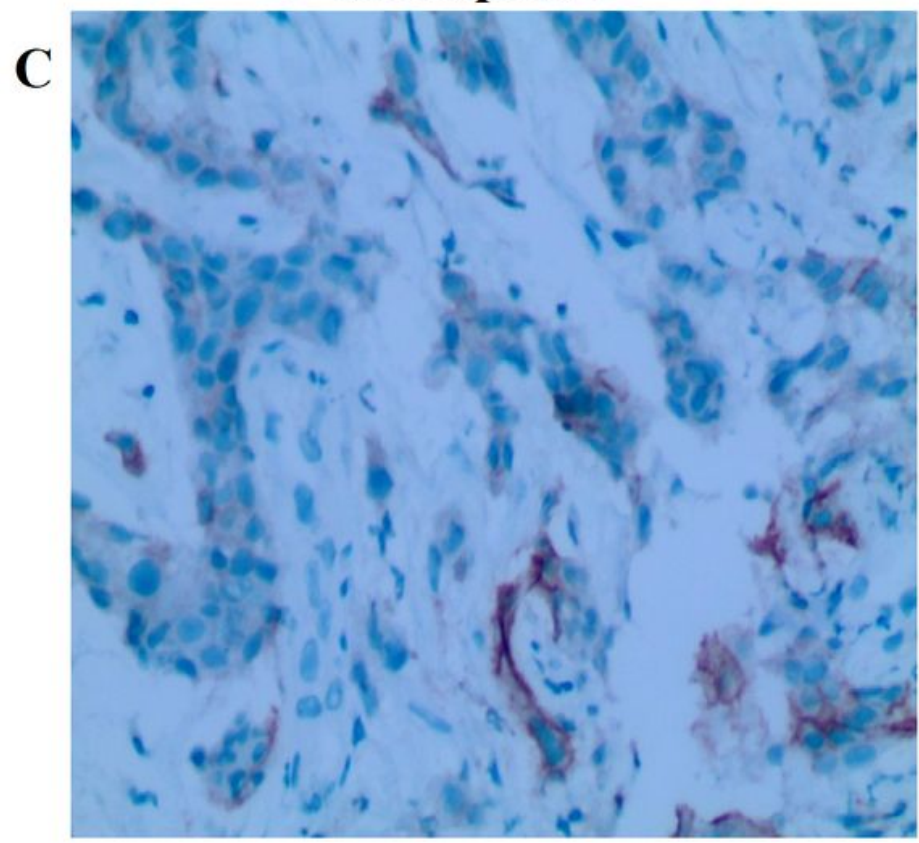

\section{Figure 3}

(Patient 3) A. Contrast-enhanced chest CT prior and best response to treatment. B. Hematoxylin and eosin-stained section of lung lesion biopsy specimen. C. PD-L1 protein expression assessed by immunohistochemistry. 